\title{
Inferring Gene Network from Candidate SNP Association Studies Using a Bayesian Graphical Model: Application to a Breast Cancer Case-Control Study from Ontario
}

\author{
Sohee Kanga, b Sevtap Savas ${ }^{c}$ Hilmi Ozcelik $^{c}$ Laurent Briollais $^{\mathrm{b}, \mathrm{d}}$ \\ ${ }^{a}$ Department of Computer and Mathematical Sciences, University of Toronto Scarborough, Scarborough, Ont., \\ bProsserman Centre for Health Research, and ' Fred A. Litwin Centre for Cancer Genetics, Lunenfeld-Tanenbaum \\ Research Institute, and d Dalla Lana School of Public Health, University of Toronto, Toronto, Ont., Canada
}

\section{Key Words}

Gene network analysis · Candidate SNP association .

Bayesian graphical model · Breast cancer · Case-control study

\begin{abstract}
Background/Aims: Gene network analysis can be a very valuable approach for elucidating complex dependence between functional SNPs in a candidate genetic pathway and for assessing their association with a disease of interest. Even when the number of SNPs evaluated is relatively small $(<20)$, the number of potential gene networks induced by the SNPs can be very large and the contingency tables representing their joint distribution very sparse. Methods: In this paper, we propose a Bayesian model determination for gene network analysis using decomposable discrete graphical models combined with Reversible Jump Markov chain Monte Carlo. We show the application of this approach in a study of 13 SNPs in the DNA repair pathway and their association with breast cancer from a case-control study conducted in Ontario, Canada. Results: The strength of associations among the SNPs and between the SNPs and the disease status is evaluated by computing the posterior probability of any pair of variables. The corresponding gene network is re-
\end{abstract}

constructed by retaining pair-wise associations with the highest posterior probabilities. In our real data analysis, we found evidence for a particular association between one SNP in the gene POLL and the disease status and also several interesting patterns of association between the SNPs themselves. Conclusion: This general statistical framework could serve as a basis for prioritizing genes and SNPs that play a major role in breast cancer etiology and to better understand their complex interactions in a specific genetic pathway.

(c) 2014 S. Karger AG, Basel

\section{Introduction}

Biological networks or biological systems represent knowledge about molecules, processes and their interactions, i.e. the cellular wiring diagram. Biological researchers use network maps to design and analyze experiments and to make predictions about the behavior of biological systems. The functional interactions between genes, molecules and/or other biological processes are also fundamental to understanding the joint basis of disease etiology. Network information is, unfortunately, extremely difficult to infer and biologists generally use fragmented

\section{KARGER}

E-Mail karger@karger.com

www.karger.com/hhe
(C) 2014 S. Karger AG, Basel

0001-5652/14/0784-0140\$39.50/0
Dr. Laurent Briollais

Lunenfeld-Tanenbaum Research Institute, Mount Sinai Hospital 60, Murray Street

Toronto, ON M5T 3L9 (Canada)

E-Mail laurent@lunenfeld.ca 
and incomplete information. To this extent, statistical methods such as graphical models can be very useful to evaluate complex dependence patterns in a given gene network.

Indeed, graphical models have been one of the most efficient statistical tools used in the last 20 years for the analysis of complex structured data [1]. Graphical models provide a probabilistic framework for making inference and representing the knowledge that we have about these complex structured data. In biological research and more particularly in the emerging '-omics disciplines' such as genomics, proteomics, metabolomics, or transcriptomics, data are often generated from complex high-throughput experiments and from complex experimental designs. Graphical models can represent these complex biological problems, leading to relatively simple and tractable computational algorithms to obtain the quantities of interest. The graph underlying the graphical model consists of a set of vertices and a set of edges linking some of the vertices. In biological studies, the vertices represent the variables such as mRNA expression levels, protein levels, environmental conditions, genotypes, and phenotypes.

The ability to model complex dependency structures makes graphical models particularly attractive for gene network analysis in complex diseases such as breast cancer. Indeed, the factors underlying genetic susceptibility to breast cancer continue to remain largely unknown. To date, the prediction of breast cancer susceptibility in high-risk women has been dependent upon genetic testing for the presence of high-penetrant mutations, which deleteriously impact upon the function of BRCA1 and BRCA2 and other specific genes. An important caveat, however, is that these mutations are rare and account for only $10-20 \%$ of familial and $5-10 \%$ of all breast cancer cases in the population. Beside these mutations, it is likely that interactions (epistasis) among commonly occurring SNPs explain the altered (increased or decreased) disease susceptibility [2]. A recent paper demonstrated how information on gene network inferred from expression data could provide a very significant tool for predicting breast cancer-related outcomes [3].

Despite its obvious advantages, the use of Bayesian graphical models remains relatively rare in genetic research, in particular its application to discrete data such as genotype data. This could be partly explained by the computational difficulties in fitting graphical models to complex structured data. The early applications of graphical models in genetic research can be found in peeling methods of Elston and Stewart [4] and Thompson et al. [5]. Lauritzen and Sheehan [6] give an overview and dis-

Gene Network Analysis in Association Studies cussion of graphical modeling methods in genetics and related fields. Several authors have also used graphical modeling in multipoint linkage analysis [7-9].

The application of graphical models to discrete data, such as SNPs, remains relatively rare. Among the few examples, Thomas and Camp [10] proposed to use graphical models to study the patterns of allelic association between genetic markers in a small chromosomal region. Their work focused on decomposable graphical models in the frequentist framework and used simulated annealing for model fitting. However, their application to 25 loci within a known gene raised some questions, because the patterns of associations they found did not follow any natural physical ordering and the results were very sensitive to the annealing parameter. A more recent approach shows the interest of Bayesian graphical models in the context of genome-wide association studies [11]. Unlike Thomas and Camp [10], these authors proposed a fully Bayesian approach based on the work of Madigan et al. [12], where prior knowledge about linkage disequilibrium around each marker can be incorporated. The model fitting used a Markov chain Monte Carlo (MCMC) algorithm that yields samples from the posterior probability. Inference was based on model averaging. They used decomposable graphical models where their clique definition was restricted to SNPs physically close to each other, ignoring the complex nature of association patterns in genome-wide association studies. Additional work on the application of probabilistic graphical models to genetic associations were also reported using either the Bayesian framework $[13,14]$ or the frequentist framework $[15,16]$.

In this paper, our gene network analysis focuses on SNPs implicated in DNA repair processes, which have long been known to be associated with certain inherited diseases such as premature aging syndromes and cancer [17-19]. To date, approximately 100 genes functioning within 5 DNA damage recognition and repair pathways have been identified $[20,21]$. In humans, extensive genetic variation occurs in DNA repair genes [21, 22]. It has been hypothesized that genetic variation in DNA repair genes may modify the individual and population cancer risk [21]. In fact, experimental evidence indicates that there are differences in DNA repair capacities among individuals, and a reduced DNA repair capacity is associated with an increased cancer risk [22, 23]. We propose a Bayesian graphical model approach to study gene networks induced by SNPs within this pathway and their association with breast cancer. We selected 13 SNPs that are involved in the DNA repair pathway and fitted decomposable graphical models to these data. The main inter- 
ests of our study lie in the search for genetic associations with breast cancer and in the study of specific patterns of SNP associations that could describe their functional relationships.

The paper is organized as follows: in the next section, we review some theoretical results on graphical models for discrete data. Then, we introduce the Bayesian graphical modeling approach, and we describe the Reversible Jump Markov chain Monte Carlo (RJMCMC) sampler to fit this model. We proceed by presenting a real application of the method to a breast cancer data set. Later, the results and future perspectives are discussed. Finally, some technical details about the method are given in the appendices.

\section{Graphical Modeling for Discrete Data}

Graphical modeling is a form of multivariate analysis that uses graphs to represent models [24]. A graph $G=(V$, $E$ ) is a mathematical object consisting of a finite set $V$ of vertices (or nodes) and a finite set $E$ of edges (or arcs) between these vertices. The vertices in the graph correspond to random variables that can be discrete or continuous, and the edges in the graph describe the dependency (or conditional independency) that hold for the joint distribution of variables. There are two major types of graphical models: the undirected and the directed graphical models. We will focus on undirected graphs whose edges do not have directions. In the following, we refer to $C(G)$ as the collection of cliques of $G$ and to $S(G)$ as a system of separators in a perfect ordering of such cliques.

Discrete graphical models describe the relation between a set of $k=|V|$ discrete random variables, $X_{v}=\left(X_{v}\right)_{v}$ $\in V$, each of which takes values (levels) in $I_{v}$. The data is collected in a contingency table with cells indexed by the combination of levels for the $|V|$ variables. Let $I$ be the set of all cells in the table such that $I=\prod_{v \in V} I_{v}$, where $I_{v}$ is the set of levels of factor $v$. We define cell probabilities as $\theta^{G} \in M(G)$, where $M(G)$ is a Markov family of probability distributions with respect to a graph $G$, i.e. satisfying all conditional independence that can be read off from graph $G$. When the graph is decomposable, the arbitrary distribution $\theta^{G}$ is determined by the marginal probability tables $\theta_{C}^{G}=\left(\theta_{C}^{G}\left(i_{C}\right)\right)_{i_{c} \in I_{c}}, C \in C(G)$, with elements $\theta_{C}^{G}\left(i_{C}\right)=$ $P\left\{X_{C}=i_{C}\right\}$ as in the following:

$$
\theta^{G}(i)=\frac{\prod_{C \in C(G)} \theta_{C}^{G}\left(i_{C}\right)}{\prod_{S \in S(G)} \theta_{S}^{G}\left(i_{S}\right)} .
$$

Note that $i_{C} / i_{S}$ indicates a cell of the marginal contingency table corresponding to the variables in $C / S$, which is a subset of $V$ corresponding to a clique or to a separator.

Data for contingency tables are collected using various sampling schemes. Here, we will consider multinomial sampling, where the total number of observations is fixed but cell counts are otherwise random. In this case, if $x_{v}^{(n)}$ are the observed values of a random sample of $n$ observations, the likelihood $L\left(\theta^{G}\right)=p\left(X_{v}^{(n)}=x_{v}^{(n)} \mid \theta, G\right)$ can be written as follows:

$$
L\left(\theta^{G}\right)=\frac{\prod_{C \in C} p\left\{X_{C}^{(n)}=x_{C}^{(n)} \mid \theta_{C}^{G}\right\}}{\prod_{S \in S} p\left\{X_{S}^{(n)}=x_{S}^{(n)} \mid \theta_{S}^{G}\right\}}=\frac{\prod_{C \in C} \prod_{i_{A} \in I_{C}}\left(\theta_{A}^{G}\left(i_{A}\right)\right)^{n_{A}\left(i_{A}\right)}}{\prod_{S \in S_{i_{A}} \in I_{S}}\left(\theta_{A}^{G}\left(i_{A}\right)\right)^{n_{A}\left(i_{A}\right)}},
$$

where

$$
n_{A}\left(i_{A}\right)=\sum_{j: j_{A}=i_{A}} n(j)
$$

is the observed count in cell $i_{A}$ of the marginal table of $X_{A}$.

A notable property of a decomposable graphical model is that the joint density (likelihood) can be factorized in terms of clique marginal densities. It is also possible to get the explicit form of the MLEs [25]. The expressions above are valid even for high-dimensional contingency tables, where each discrete random variable can have any number of possible values. An example of a decomposable graph $G$ is given in the online supplementary material ('A small example of graphical model representation', see www.karger.com/doi/10.1159/000365923 for all online suppl. material). In this case, we have a three-way contingency table, where each variable is binary and the joint distribution of the 3 binary variables satisfies the Markov property.

\section{Bayesian Graphical Modeling for Discrete Data}

\section{Hyper-Dirichlet Prior Distribution for Bayesian \\ Learning}

There are 2 main aspects in the statistical inference with Bayesian graphical models: one is to estimate the unknown parameters $\theta^{G}$ using the information available, and the other is to obtain the graphs or graphical models that are best supported by the data and available prior information. The choice of prior distribution for the model parameters may have a considerable impact on posterior model probabilities. Dawid and Lauritzen [26] demonstrated that there exists a unique hyper-Dirichlet distribution for $\theta$ over decomposable graphical models. For 
each clique $C \in C$, let $D\left(\lambda_{C}\right)$ denote the Dirichlet distribution for $\theta_{C}$ with density

$$
\pi\left(\theta_{C} \mid \lambda_{C}\right) \propto \prod_{i_{C} \in I_{C}} \theta_{C}\left(i_{C}\right)^{\lambda_{C}\left(i_{C}\right)-1},
$$

where $\lambda_{C}\left(i_{C}\right)>0$ for all $i_{C} \in I_{C}$.

In order to construct a hyper-Dirichlet distribution, we must satisfy the condition of consistency of the matrices of cell probabilities, and furthermore, the hyperparameters $\lambda_{C}$ are constrained in order to satisfy the hyperconsistency condition of the corresponding distribution $D\left(\lambda_{C}\right)$, defined as:

$$
\lambda_{C \cap D}\left(i_{C \cap D}\right)=\sum_{j_{C}: j \subset \cap D=i_{C \cap D}} \lambda_{C}\left(j_{C}\right)=\sum_{j_{D}: j \subset \subset D=i_{C \cap D}} \lambda_{D}\left(j_{C}\right) .
$$

This constraint is satisfied by assigning a Dirichlet distribution on parameter $\theta$, corresponding to the complete graph, and by obtaining the laws on the cliques by marginalization. Alternatively, as suggested by Dawid and Lauritzen [26], we can take:

$$
\lambda(i)=\frac{\prod_{C \in C_{G}} \lambda_{C}\left(i_{C}\right)}{\prod_{S \in S_{S}} \lambda_{S}\left(i_{S}\right)} .
$$

We can also find the closed form expression for the marginal likelihood (normalizing constant) (see details in Dawid and Lauritzen [26]):

$$
p_{A}\left(x^{(n)}\right)=\frac{\Gamma\left(\lambda_{0}\right)}{\Gamma\left(\lambda_{0}+n\right)} \prod_{i_{A} \in I_{A}}\left(\frac{\Gamma\left(\lambda_{A}\left(i_{A}\right)+n_{A}\left(i_{A}\right)\right)}{\Gamma\left(\lambda_{A}\left(i_{A}\right)\right)}\right) .
$$

Regarding the values of the hyperparameters, which can be interpreted as hypothetical marginal data counts, one possibility is to assign $\lambda(i)=1 / 2$, following Jeffrey's prior for multinomial sampling, or $\lambda(i)=1$, following a uniform distribution. Another possible formulation is to consider a hierarchical prior which provides more flexibility, for instance letting

$$
\lambda_{0}=\sum_{i \in I} \lambda(i)=\sum_{i_{C} \in I_{C}} \lambda_{C}\left(i_{C}\right)
$$

be a random variable for which to assign a prior distribution.

Following Tarantola [27], we employed a hyperDirichlet distribution with hierarchical prior for the choice of the prior in this work, to simplify the calculations and to control the size of the graphs generated from the MCMC samplers.

Gene Network Analysis in Association

\section{Bayesian Model Averaging}

In order to select the best graphs, we applied 2 important Bayesian principles. First, as proposed in Madigan and Raftery [28], models with a low posterior probability compared to the highest posterior probability model are discarded. This choice drastically reduces the size of the target model space. We denote the reduced model space $\mathbf{A}^{\prime}$ with:

$$
\mathbf{A}^{\prime}=\left\{G_{k}: p\left(G_{k} \mid x\right) \geq c \times \max _{l} p\left(G_{l} \mid x\right)\right\},
$$

where $c \in(0,1)$. Different choices of $c$ are possible. For example in our applications, we deleted those graphs which appear $\leq 5$ times after 200,000 iterations of the MCMC algorithms. So $c$ was chosen accordingly. Second, the evidence in favour of association either between each SNP and the disease or between the SNPs themselves is based on model averaging [28-30] and computing the posterior edge probability. If $\Delta$ represents a particular edge for the association between a SNP and the disease or between the SNPs themselves, then its posterior given data $x$ is:

$$
p(\Delta \mid x)=\sum_{G_{k} \in \mathcal{A}^{\prime}} p\left(\Delta \mid G_{k}, x\right) p\left(G_{k} \mid x\right) .
$$

This is an average of the posterior distributions under each model in set $\mathbf{A}^{\prime}$, weighted by their posterior model probability. To compute the sum in equation 8 , we used a Monte Carlo integration and derived the edge posterior probability given the data as:

$$
p(\Delta \mid x)=E_{p\left(G_{k} \mid x\right)}\left[p\left(\Delta \mid G_{k}, x\right)\right] \approx \frac{1}{\left|\mathcal{A}^{\prime}\right|} \sum_{G_{k} \in \mathcal{A}^{\prime}} I\left(\Delta \mid G_{k}, x\right),
$$

where $I($.$) is the indicator function, equal to 1$ if graph $G_{k}$ contains an edge for the association of interest $\Delta$ and 0 otherwise. We decided to keep all edges with a posterior probability $\geq 0.2$ and to assess the accuracy of our inference procedure to recover the true graph in our simulation study (see Validation of the Method by Simulation Studies below).

\section{Implementation of Model Selection}

For large tables, the number of possible models becomes too large to be evaluated exhaustively and it becomes difficult to choose a best model. Dawid and Lauritzen [26] proposed an explicit expression for comparing 2 decomposable models that differ by the presence of one edge, using the Bayes factor, but this calculation becomes unfeasible for large tables. Therefore, alternative strategies are needed when investigating a large number of models. Here, we present an approach for Bayesian 
model determination for decomposable discrete graphical models which relies on MCMC algorithms to sample over the space of possible discrete graphs. We apply the RJMCMC algorithm of Green [31], which updates the model and the corresponding parameters at each step. This algorithm was used for example by Dellaportas and Forster [32] for hierarchical, graphical or decomposable log-linear models for high-dimensional contingency tables.

The RJMCMC Sampler

A general difficulty with MCMC approaches is to make an inference about transdimensional models, i.e. models whose structure is associated with a different set of parameters. In general, these sets will be of different sizes and as we move from one model to another, the parameter space of interest changes dimension. Such jumps are not well accommodated by MCMC without significant modification [33]. As an alternative, the RJMCMC sampler [31] allows us to move inside the space of models and the corresponding parameters such that we move from $\left(G, \theta^{G}\right)$ to $\left(G^{\prime}, \theta^{G^{\prime}}\right)$. For graphical models, it involves 2 steps: the first step is to propose the new graphical structure as adding or deleting an edge, and the second step is to update the matrix of cell probabilities given the new graphical structure. The proposal distribution for the move from $\left(G, \theta^{G}\right)$ to $\left(G^{\prime}, \theta^{G^{\prime}}\right)$ can be written as:

$$
\operatorname{Prop}\left(G, \theta^{G} \rightarrow G^{\prime}, \theta^{G^{\prime}}\right)=q\left(G \rightarrow G^{\prime}\right) \times q_{G G^{\prime}}\left(\theta^{G}, \theta^{G^{\prime}}\right),
$$

where $q\left(G \rightarrow G^{\prime}\right)$ is the probability to attempt a move from graph $G$ to $G^{\prime}$ and $q_{G G}\left(\theta^{G}, \theta^{G^{\prime}}\right)$ is the conditional probability to move from parameters $\theta^{G}$ to $\theta^{G \prime}$ given the new graph structure.

Following Tarantola's approach [27], the 2 steps of the RJMCMC algorithm can be summarized as follows.

\section{Update of the G Move}

The rationale for this step is to keep the distribution of the cliques that are not involved in the change constant, and to assign a distribution only on the new clique, but it has to be consistent with the distribution of the former cliques. For example, let's consider the addition of an edge between two vertices as the next move. This will generate a new clique with separator $S$. Given a graph $G$ with the collection of parameters $\left(\theta_{C}^{G}, C \in C(G)\right)$, we propose to move to a new graph $G^{\prime}$ with the new collection of parameters $\left(\theta_{C}^{G^{\prime}}, C \in C\left(G^{\prime}\right)\right)$. The second collection of parameters now includes the distribution of the newly created clique. After generating the distribution of the pa- rameters for the newly generated clique, the proposed addition of an edge move is accepted with a probability equal to:

$$
\alpha=\min \left\{1, R_{a}\right\},
$$

where

$$
R_{a}=\frac{p\left(G^{\prime}, \theta^{G \prime} \mid x\right)}{p\left(G, \theta^{G} \mid x\right)} \times \frac{r_{D}\left(G^{\prime}, \theta^{G^{\prime}}\right)}{r_{A}\left(G, \theta^{G}\right) q(z)} \times|J|=R_{\text {post }} \times R_{\text {prop }} \times|J| .
$$

Here, $r_{A}\left(G, \theta^{G}\right)$ is the probability of adding an edge and $r_{D}\left(G^{\prime}, \theta^{G^{\prime}}\right)$ is the reverse move, which is the probability of deleting an edge. The function $q(z)$ is the probability distribution of some auxiliary variables considered in order to satisfy the dimension-matching condition (i.e. the dimension of the current graph plus the number of variables required to map to an appropriate set of parameters for the new graph must be preserved between each possible jump and the reverse move [33]). Note that since $p\left(G, \theta^{G} \mid x\right) \propto p\left(x \mid G, \theta^{G}\right) p\left(\theta^{G} \mid G\right) p(G), R_{\text {post }}$ can be written as:

$$
R_{\text {post }}=\frac{p\left(x \mid G^{\prime}, \theta^{G^{\prime}}\right)}{p\left(x \mid G, \theta^{G}\right)} \times \frac{p\left(\theta^{G^{\prime}} \mid G^{\prime}\right)}{p\left(\theta^{G} \mid G\right)} .
$$

\section{Update of the $\theta^{G}$ Move}

The principle of this second step is to update the matrix of cell probabilities given the new graphical structure generated in the first step. This procedure does not change the set of cliques and separators but modifies the set of probabilities associated with them. The second step also helps the chain to move more efficiently inside the parameter space improving the mixing of the chain. Following Tarantola [27], one clique $C \in C(g)$ is randomly selected and its elements $\theta_{C}^{G}\left(i_{C}\right)$ are perturbed by adding a uniform random variant $u_{i} \sim \operatorname{Uniform}(-\delta, \delta)$. Since the update is only involving the parameters of clique $C$, the proposed move is accepted with a probability of $\alpha=$ $\min \left\{1, R_{a}\right\}$, where

$$
R_{a}=\frac{p\left(x \mid G, \theta_{C}^{G^{\prime}}\right) \pi\left(\theta_{C}^{G^{\prime}} \mid G^{\prime}\right)}{p\left(x \mid G, \theta_{C}^{G}\right) \pi\left(\theta_{C}^{G} \mid G\right)} \times R_{\text {prop }},
$$

and the proposal ratio $R_{\text {prop }}$ is equal to 1 since the proposal distribution is symmetric.

\section{Hierarchical Prior}

To control the robustness of the graphical model generated by the RJMCMC sampler, we added one more hierarchy to the model by introducing a random quantity 
Table 1. Description of the 13 selected DNA repair SNPs

\begin{tabular}{|c|c|c|c|c|c|c|c|c|}
\hline Gene name & SNP & $\begin{array}{l}\text { Nucleotide } \\
\text { change }\end{array}$ & $\begin{array}{l}\text { PolyPhen } \\
\text { prediction }\end{array}$ & $\begin{array}{l}\text { Function } \\
\text { (literature) }\end{array}$ & $\begin{array}{l}\text { Minor allele } \\
\text { frequency } \\
\text { (nucleotides) }\end{array}$ & $\begin{array}{l}\text { Odds } \\
\text { ratio }^{\text {a }}\end{array}$ & $\begin{array}{l}\mathrm{p} \\
\text { value }^{\mathrm{a}}\end{array}$ & $\log \mathrm{BF}^{\mathrm{b}}$ \\
\hline BRCA1 & rs16941 & E1038G & benign & $\mathrm{n} / \mathrm{a}$ & 0.57 (G/A) & 1.04 & 0.79 & -1.15 \\
\hline ERCC6 & rs2228527 & $\mathrm{R} 1213 \mathrm{G}$ & benign & $\mathrm{n} / \mathrm{a}$ & 0.37 (G/A) & 1.26 & 0.12 & 0.23 \\
\hline EXO1 & rs9350 & P757L & probably damaging & $\mathrm{n} / \mathrm{a}$ & $0.35(\mathrm{~T} / \mathrm{C})$ & 0.87 & 0.39 & -0.81 \\
\hline P53 & rs1042522 & P72R & benign & functional & $0.42(\mathrm{C} / \mathrm{G})$ & 0.98 & 0.91 & -1.17 \\
\hline POLL & rs3730477 & R438W & benign & $\mathrm{n} / \mathrm{a}$ & $0.42(\mathrm{~T} / \mathrm{C})$ & 0.73 & 0.034 & 1.87 \\
\hline POLQ & rs1381057 & Q2513R & benign & $\mathrm{n} / \mathrm{a}$ & $0.50(\mathrm{~T} / \mathrm{C})$ & 1.09 & 0.56 & -1.01 \\
\hline PRIM1 & rs2277339 & D5A & probably damaging & $\mathrm{n} / \mathrm{a}$ & $0.19(\mathrm{C} / \mathrm{A})$ & 1.32 & 0.12 & -0.30 \\
\hline RAG1 & rs2227973 & K820R & benign & $\mathrm{n} / \mathrm{a}$ & $0.19(\mathrm{G} / \mathrm{A})$ & 1.23 & 0.25 & -0.51 \\
\hline WRN & rs1800391 & M387I & possibly damaging & $\mathrm{n} / \mathrm{a}$ & $0.16(\mathrm{~A} / \mathrm{G})$ & 1.01 & 0.99 & -1.15 \\
\hline
\end{tabular}

${ }^{a}$ Odds ratio and $\mathrm{p}$ value for a single SNP association.

$\mathrm{b}$ The log Bayes Factor was calculated assuming a proportional prior table with a sample size equal to $10 \%$ of the original sample size.

$\lambda_{0}$ which is the total prior precision [27]. Assuming $\lambda_{0}$ and $G$ are independent, we assign the prior distribution of $\lambda_{0}$ as Gamma distribution with a mean of $a$ and a variance of $a b$,

$$
p\left(\lambda_{0}\right) \propto \lambda_{0}^{(\mathrm{a} / \mathrm{b})-1} \exp \left(-\lambda_{0} / \mathrm{b}\right),
$$

where $a>0$ and $b>0$. Once the new total prior precision $\lambda_{0}^{\prime}$ is assigned, the cell parameters $\lambda^{\prime}(i), i \in I$ are obtained as $\lambda^{\prime}(\mathrm{i})=\lambda^{\prime}{ }_{0} /|I|$.

The MCMC algorithm now involves an extra step which is to update the random parameter $\lambda_{0}$. For example, a change in $\lambda_{0}$ is accepted with a probability of $\alpha=$ $\min \left\{1, R_{a}\right\}$, where

$$
R_{a}=\frac{p\left(\theta \mid G, \lambda_{0}^{\prime}\right) p\left(\lambda_{0}^{\prime}\right)}{p\left(\theta \mid G, \lambda_{0}\right) p\left(\lambda_{0}\right)} .
$$

\section{Application to a Case-Control Genetic Association Study of Breast Cancer}

In this study, we searched for possible associations among several SNPs in the DNA repair pathway and breast cancer and between the SNPs themselves. A total of 13 SNPs from 13 DNA repair genes were genotyped in 398 breast cancer cases selected from the Ontario Family Breast Cancer Registry (OFBCR) [34] and 372 agematched population-based controls. All of them have been shown to be functionally important or are good candidates to be associated with breast cancer based on previous association findings (table 1). The functionally characterized SNPs included for example OGG1-S326C, reported to have a lower capacity to repair mutations induced by 8-hydroxyguanine [35], and p53-P72R, whose proline variant is associated with a lower antiproliferative effect of p53 protein [36]. In addition, a XRCC1-R280H substitution results in a defective XRCC1 protein that does not efficiently localize to a damaged site in the chromosome for repair [37]. This causes a reduction in DNA repair capacity of the cell. For the remaining SNPs, we used the bioinformatic software PolyPhen [38] to predict their functional effects. As a result, EXo1-P757L, IGHMBP2-T671A, PRIm1-D5A, WRN-M387I, and XRCC1-R280H were predicted to affect protein functions. A description of these SNPs is given in table 1 . These SNPs are all bi-allelic and are relatively common in the general population with a minor allele frequency $>5 \%$. For each SNP, we have 3 possible genotypes that were further dichotomized using either a dominant or recessive genetic model to simplify the computations. The best model for each SNP was chosen using the AIC criterion. The binary variable $Y_{i} \in\{0,1\}$ is the disease status for individual $i$ with observed value $y_{i}=0$ for a control and $y_{i}=$ 1 for a breast cancer case. None of the marginal associations of these SNPs with the disease status were significant at a 5\% significance level except those of the SNP in 
Fig. 1. Graph generated by the RJMCMC sampler with hyperparameter $a=1$.
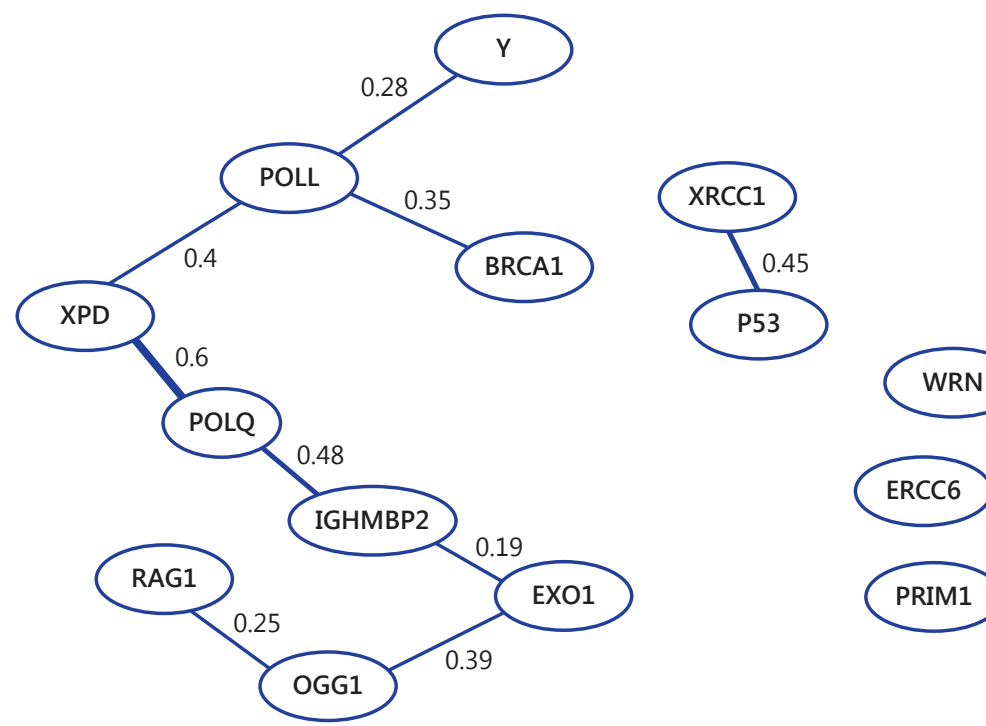

PRIM1

the gene POLL $(\mathrm{OR}=1.87, \mathrm{p}=0.034$; table 1$)$. The log Bayes Factors, assessing evidence for SNP association, were computed with the $R$ package 'LearnBayes'. We found that only the SNP in the POLL gene shows strong evidence (i.e., $1 \leq \log \mathrm{BF} \leq 2$ ) against the null hypothesis of no association.

Next, our goal was to use the posterior probabilities from the Bayesian graphical models to infer the gene network induced by the 13 SNPs and to assess the importance of SNP-disease associations and SNP-SNP associations in this data. In the following, we use gene labels to refer to a particular SNP for ease of notation.

\section{Results from the RJMCMC Sampler}

Here, we describe the results from the RJMCMC sampler. We also assess its sensitivity to the prior specification. There are $2^{91}$ possible undirected graphs. The RJMCMC sampler approximately reached convergence after 200,000 iterations of the chains with a burn-in period of 20,000 iterations. For every 200th iteration, we traced the number of edges in each graph and plotted the corresponding cumulative mean (online suppl. fig. S1 in 'Diagnostic plot for the number of edges'). The RJMCMC sampler explored more frequently graphs with $7,8,9$, and 10 edges ( $71 \%$ of all graphs), and the cumulative mean of the number of edges indicates a stability of the chain
Table 2. Probability of edge inclusion from RJMCMC with different hyperparameter values

\begin{tabular}{llll}
\hline Edge & $a=1$ & $a=32$ & $a=64$ \\
\hline [XPD, POLQ] & 0.60 & 0.67 & 0.69 \\
[POLQ, IGHMBP2] & 0.48 & 0.51 & 0.55 \\
[XRCC1, P53] & 0.45 & 0.46 & 0.55 \\
[XPD, POLL] & 0.40 & 0.49 & 0.42 \\
[OGG1, EXO1] & 0.39 & 0.48 & 0.47 \\
[POLL, BRCA1] & 0.35 & 0.38 & 0.45 \\
[POLL, Y] & 0.28 & 0.38 & 0.46 \\
[RAG1, OGG1] & 0.25 & 0.28 & 0.29 \\
[IGHMBP2, EXO1] & - & 0.24 & 0.25 \\
[XPD, EXO1] & - & 0.23 & 0.25 \\
[RAG1, BRCA1] & - & 0.20 & - \\
[XPD, Y] & - & - & 0.22 \\
[RAG1, PRIM1] & - & - & 0.21 \\
\hline
\end{tabular}

around 9 edges. We deleted the graphs that occurred $<5$ times throughout the iterations (see Bayesian Model Averaging above). The corresponding averaged graph is given in figure 1.

We also assessed the sensitivity of the graph structure generated by the RJMCMC sampler to the specification of the priors. We chose hyperparameter values for the prior distribution of $\lambda_{0}$ as Gamma distribution with a mean of $a=1, a=32$ and $a=64$ and a variance of $a b=$ 0.1 in both cases. The convergence plots show that the 
Fig. 2. Union of the three graphs generated by the RJMCMC sampler with hyperparameter $a=1,32$ and 64 . The red line represents the common edges between any two graphs. The green lines indicate the unique edges for the three graphs. The blue lines are common edges across all three graphs.

Fig. 3. Intersection of the three graphs when hyperparameter $a=1,32$ and 64 .
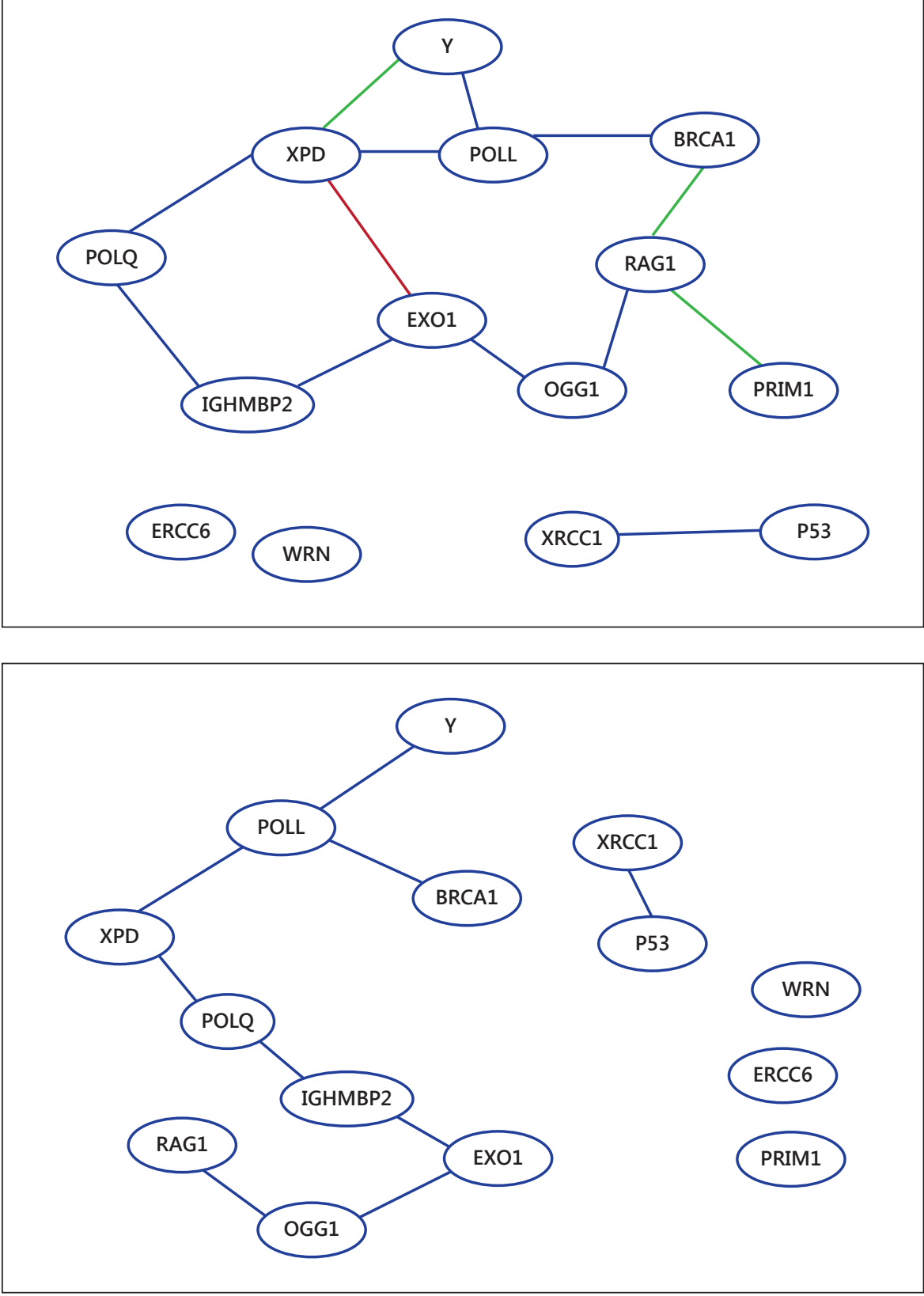

graphs have approximately 11 and 12 edges, when $a=32$ and $a=64$, respectively. In table 2, we listed the corresponding 13 highest posterior probabilities of associations between the vertices. We generated the three graphs corresponding to the three different choices of hyperparameters, summarizing the results in one union graph (fig. 2).

Finally, to summarize the main results from the RJMCMC sampler, we plotted the intersection of the three graphs (i.e., with $a=1,32$ and 64) in figure 3. Based on figure 3 , we can also make several interesting remarks. The results of primary interest concern variable $Y$ (the disease status) and its links with the SNP variables. There is evidence for a direct relationship between the disease status and the SNP in the gene POLL. The posterior probability for this edge varies between 0.28 and 0.46 . There is also a consistent structure of dependencies involving the disease status and the three SNPs present in the genes 
Fig. 4. Conditional dependence between three SNPs and the disease status $Y$.
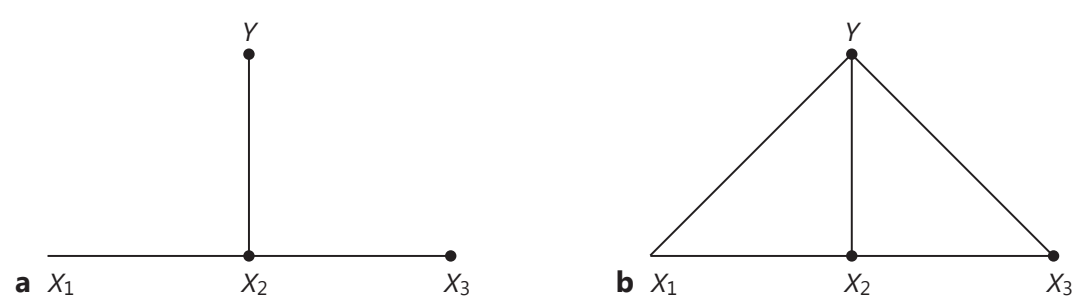

BRCA1, POLL and XPD. The disease status is directly linked to the SNP in the gene POLL and indirectly linked to the SNPs in the genes XPD and BRCA1 through POLL. We also noted some higher-order dependencies between the disease status and the SNPs in the genes EXO1, POLQ, OGG1, and IGHMB2. The results of secondary interest regard the pattern of dependencies between the SNPs themselves. The algorithm shows evidence for important links including [XPD, POLQ], [POLQ, IGHMBP2], and [XRCC1, P53] (the lowest posterior probability is 0.6 , 0.48 and 0.45 , respectively, for each link). In addition, we also found a long chain of association [Y-POLL-XPDPOLQ-IGHMBP2-EXO1-OGG1-RAG1]. Some edges have some high posterior probabilities for certain choices of hyperparameters but were not included in figure 3 because they were not consistently found after changing the hyperparameter values. Finally, we also identified an association between two SNPs [P53, XRCC1] that was not linked to the disease status.

For our analyses, we used a modification of the $M C^{3}$ Fortran program written by Tarantola [27] linking to a NAG library. All computations were done on a SUN workstation with an Intel dual-core Opteron 2.4-GHz processor running the CentOS4.4 operating system. The running time varied depending on the specification of the hyperparameters (higher values increased the running time).

\section{Validation of the Method by Simulation Studies}

To assess the properties of our Bayesian graphical model approach, we simulated data sets that mimic genetic associations between three SNPs $\left(X_{1}, X_{2}, X_{3}\right)$ and a disease status $(Y)$ under the two scenarios represented in figure 4 . We simulated 20 data sets of 400 cases and 400 controls each. The distribution of the four discrete variables in the graph was generated from a multinomial dis-
Table 3. Summary of edge inclusion probabilities for different values of $\theta$

\begin{tabular}{lll}
\hline Edge & \multicolumn{2}{l}{ Probability of inclusion } \\
\cline { 2 - 3 } & scenario 1 & scenario 2 \\
\hline$\left(X_{1}, Y\right)$ & 0.004 & 0.807 \\
$\left(X_{2}, Y\right)$ & 0.818 & 0.743 \\
$\left(X_{2}, X_{1}\right)$ & 0.999 & 0.999 \\
$\left(X_{3}, Y\right)$ & 0.139 & 0.279 \\
$\left(X_{3}, X_{1}\right)$ & 0.003 & 0.012 \\
$\left(X_{3}, X_{2}\right)$ & 0.999 & 0.999 \\
\hline
\end{tabular}

tribution, where the joint cell probabilities are given by the log-linear model: $\log P_{i j k l}=\theta_{i}^{Y}+\theta_{j}^{X_{1}}+\theta_{k^{2}}^{X_{2}}+\theta_{l}^{X_{3}}+\theta_{i j}^{Y X_{1}}+$ $\theta_{i k}^{Y X_{2}}+\theta_{i l}^{Y X_{3}}+\theta_{j k^{1} X_{2}}^{X}+\theta_{k l^{2} X_{3}}$. In scenario 1 (fig. 4a), the loglinear parameters were chosen as $\theta_{1}^{Y}=\ln (0.5), \theta_{1}^{X}=\theta_{1}^{X^{2}}+$ $\theta_{1}^{X}=\ln (0.15)=-1.90, \theta_{11}^{Y X_{1}}=\theta_{11}^{Y X_{3}}=0$ and $\theta_{11}^{Y X_{2}}=\ln (2.0)=$ 0.69 and $\theta_{11}^{X_{1} X_{2}}=\theta_{11^{X}}^{X} X_{3}=\ln (4.0)=1.39$ for the SNPs' main effects and the associations between SNPs. The minor allele frequency for the 3 SNPs was $15 \%$. In scenario 2 (fig. $4 \mathrm{~b}$ ), we chose the same parameter values with the exception $\theta_{11}^{Y X_{1}}=\theta_{11}^{Y X_{2}}=\theta_{11}^{Y X}=\ln (2.0)=0.69$. A summary of the inclusion probability for each edge of the graph is given in table 3 .

In scenario 1 , we were able to recover the graph perfectly. In scenario 2, one edge included in the graph $\left(X_{3}\right.$, $Y$ ) had an inclusion probability of $\sim 28 \%$, which is lower than the other edges of the graph. In our application, we use a threshold for the inclusion probability of $20 \%$. Based on this criterion, our simulation results show that we can also recover the graph in scenario 2 completely.

\section{Sensitivity/Specificity Analysis}

To evaluate the accuracy of our inference procedure to recover the true graph, we computed the sensitivity/specificity analysis of the Bayesian graphical models in our 
Fig. 5. ROC curves summarizing the accuracy of the inference procedure. a Simulation scenario 1. b Simulation scenario 2.

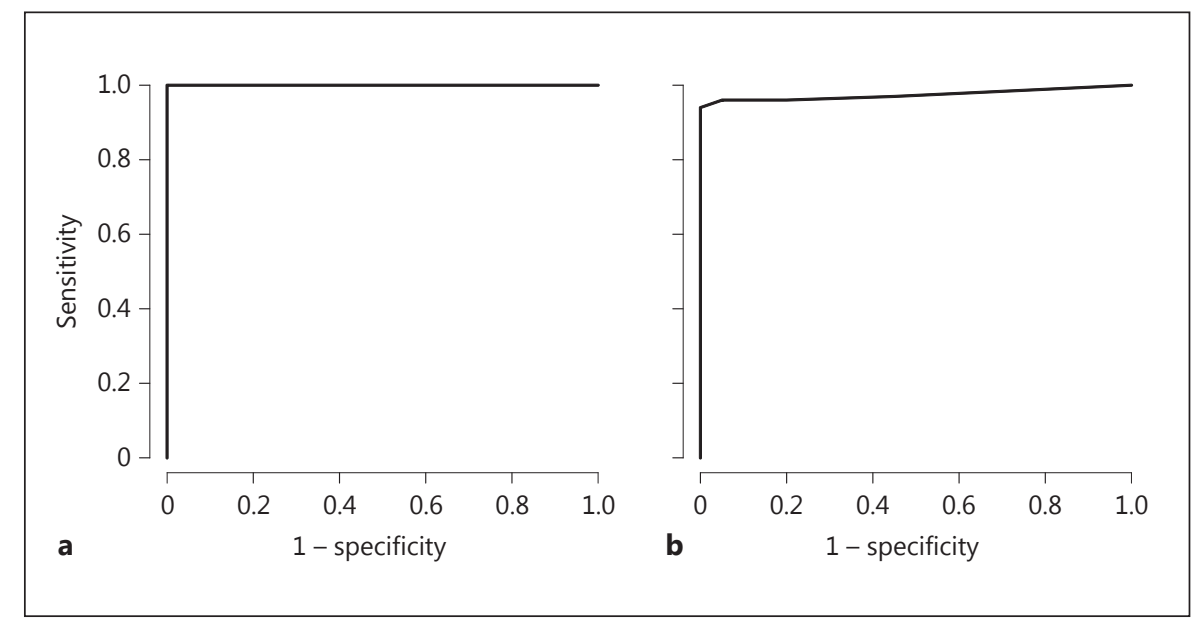

Table 4. Sensitivity and specificity of our inference procedure

\begin{tabular}{llllll}
\hline \multirow{2}{*}{$\Theta$} & \multicolumn{2}{l}{ Scenario 1} & & \multicolumn{2}{c}{ Scenario 2} \\
\cline { 2 - 3 } \cline { 5 - 6 } & sensitivity & specificity & & sensitivity & specificity \\
\hline 0 & 1 & 0 & 1 & 0 \\
0.1 & 1 & 0.67 & 0.92 & 1 \\
0.2 & 1 & 1 & 0.84 & 1 \\
0.3 & 1 & 1 & 0.80 & 1 \\
0.4 & 1 & 1 & 0.80 & 1 \\
0.5 & 1 & 1 & 0.78 & 1 \\
0.6 & 1 & 1 & 0.75 & 1 \\
0.7 & 1 & 1 & 0.73 & 1 \\
0.8 & 1 & 1 & 0.69 & 1 \\
0.9 & 0.67 & 1 & 0.63 & 1 \\
1 & 0 & 1 & 0 & 1 \\
\hline
\end{tabular}

simulation studies following the methodology presented by Husmeier [39]. In brief, let $D$ be a set of simulated data sets and $P\left(e_{i k} \mid D\right)$ be the posterior probability for edge $e_{i k}$ between nodes $i$ and $k$. Let $E(\Theta)=\left\{e_{i k} \mid P\left(e_{i k} \mid D\right)>\Theta\right\}$ denote the set of all edges whose posterior probability exceeds a given threshold $\Theta \in[0,1]$. From this set, we computed (1) the sensitivity, i.e. the proportion of recovered true edges, and (2) the complementary specificity, i.e. the proportion of erroneously recovered spurious edges. For a given threshold $\Theta$, we counted the number of true positive (TP), false positive (FP), true negative (TN), and false negative (FN) edges. We then computed the sensitivity as $\mathrm{TP} /(\mathrm{TP}+\mathrm{FN})$, the specificity as $\mathrm{TN} /(\mathrm{TN}+\mathrm{FP})$ and the complementary specificity as $1-$ specificity $=\mathrm{FP} /$ $(\mathrm{TN}+\mathrm{FP})$. We repeated this scoring procedure for several different values of $\Theta \in[0,1]$ and plotted the sensitiv-

Gene Network Analysis in Association ity scores against the corresponding complementary specificity scores. This gives the receiver operator characteristics (ROC) curve of figure 5 and corresponding table 4 . A larger area under the ROC curve indicates a better performance. Our analyses showed that keeping edges with a posterior probability $>20 \%$ yields very good accuracy to recover the simulated graph in both scenarios. The area under the ROC curve was 1 and 0.97 in scenarios 1 and 2 , respectively.

\section{Concluding Remarks}

This paper demonstrates the interest of Bayesian graphical models for gene network analysis in the context of genetic association studies. Unlike classical approaches that focus primarily on marginal SNP effects and pairwise interactions, the use of graphical models allows one to model the joint effect of multiple SNPs on the disease and reveal more complex structures of potential dependencies between them. Such effects might be particularly important for identifying low-risk variants involved in complex human diseases. For illustration, our analysis on breast cancer identified an association between breast cancer and the SNP in the gene POLL (DNA polymerase lambda). There was also evidence for several pair-wise interactions between the SNPs in genes [POLL, BRCA1], [POLL, XPD], [XPD, POLQ], and [POLQ, IGHMBP2], but the SNPs involved in these interactions were conditionally independent of the disease status given the SNP in the gene POLL. We should mention that the independence of the BRCA1 variant on the disease status $(Y)$ conditional on the POLL variant is confirmed in our data by 
fitting a log-linear model for variables $Y$, POLL and BRCA1. We found that the pair-wise interaction terms were significant for [Y-POLL] $(\mathrm{p}=0.03)$ and [POLLBRCA1] $(\mathrm{p}=0.023)$ and non-significant for [Y-BRCA1] $(\mathrm{p}=0.85)$.

The interpretation of the genetic network inferred through our statistical method is complex, and it is unclear whether the conditional dependencies among SNPs or between SNPs and breast cancer in this study represent real biological functions at the gene or protein level. There is evidence, however, that in yeast $10-20 \%$ of the genetic interactions overlap with protein-protein interactions and roughly an equivalent proportion overlaps with physical interactions, while the expected proportion of random overlaps is $3 \%$ [40]. The topology of the genetic network can also inform about possible biological functions. For example, genes that cluster together through genetic interactions tend to have redundant functions. On the other hand, hub genes (i.e. those that have many connections with other genes) often belong to different clusters, tend to have distinct functions and, in general, have a high degree of pleiotropy [40]. Therefore, as POLL and XPD are connected to several other genes and to the disease status, this could suggest interesting biological functions in relation or independently of breast cancer, but this deserves further analysis. The same study from Costanzo et al. [40] suggests that the vast majority of genetic interactions occur between, rather than within, complexes and pathways, connecting those that presumably work together or buffer one another, respectively. We therefore feel that the inference of a gene network topology, through Bayesian graphical modeling, can provide further insights into the role of genetic variants on a particular biological system. The relatively sparse graphs that we found might reflect the lack of strong conditional associations in our data set and the relatively small sample size.

Another important point is whether the SNP-SNP associations detected in our analysis are related or not related to the disease or represent population-level associations, i.e. they would be present in the population independently from the disease status. Our first intent in this paper was to study general patterns of conditional independence among the SNPs and between the SNPs and the disease that could suggest interesting functional relationships. We did not focus specifically on association patterns in relation to the disease, since our modest sample size limited this investigation; instead, our rationale was really to try disentangling direct from indirect associations of several SNPs in the DNA repair pathway with the disease. Of course, further validation is warranted. The approach can also be used as a way to prioritize SNP associations more closely related to the disease status, e.g. the nodes that are in the same clique as the disease status.

From a statistical standpoint, the Bayesian framework offers several advantages over the more classical frequentist approach. First, coupled with efficient stochastic algorithms such as MCMC methods, this framework allows exploring the models' space very quickly. This is done by sampling from the joint posterior distribution of the candidate models and regression parameters to select the best models. Second, because it is very unlikely that a single model can well represent the complexity of the data, the use of the Bayesian model averaging paradigm acknowledges the high degree of model uncertainty in high-dimensional discrete data [28]. Third, sampling methods such as MCMC techniques have a better ability to deal with missing and sparse data. Finally, prior expert knowledge can be incorporated into this framework to enhance the gene network discovery when prior information is available (e.g. from protein-protein interaction network).

Although Bayesian graphical modeling offers a flexible statistical framework for gene network analysis, research is still needed in particular regarding the design of proposed moves, which in turn can improve the speed of convergence as well as the related issue of monitoring the convergence of the algorithm. An efficient construction of the proposal distribution is also needed for a faster convergence of the chain. In this paper, 14 variables have been handled by Bayesian discrete graphical modeling, considering our relatively small sample size. It is a challenge to handle more variables as the dimension increases. There is promising research by Dobra et al. [41] using a stochastic search algorithm for high-dimensional contingency tables that we may want to consider in future work. We are also planning to integrate informative prior based on expert genetic knowledge to enhance the detection of genetic associations.

Finally, our work has implicated the biological role of the genes POLL, BRCA1 and XPD (also called ERCC2) in breast cancer development in sporadic cases. While there is no information in the literature on the possible functional effect of the BRCA1 E1038G polymorphism, the K751-XPD protein [i.e. the XPD protein having the amino acid $\mathrm{K}$ (lysine) at amino acid position 751] was shown to have a higher number of chromatid aberrations and thus reduced DNA repair capacity when compared to Q751-XPD [33]. In addition, the W438-POLL protein has reduced fidelity in vitro when compared to R438POLL, increasing the mutation rate and the number of 
chromosomal abnormalities [42]. These findings suggest a potential contribution of these two polymorphisms to cancer risk through altered POLL and XPD function. The possible conditional effect of BRCA1 and XPD genetic variants on breast cancer, mediated by POLL in our graphs, could reflect the complexity of the DNA repair pathway. POLL, BRCA1 and XPD function in different DNA repair mechanisms. While XPD is a nucleotide excision repair (NER) protein, both POLL and BRCA1 are involved in double-stranded DNA break repairs. Repair of double-stranded DNA breaks are mainly mediated by two cellular mechanisms: non-homologous end joining and homologous recombination [43]. POLL and BRCA1 function in non-homologous end joining and homologous recombination, respectively. Hence, while these three proteins do not work in the same specific DNA repair mechanism, these literature findings show the importance of these proteins and their associated SNPs (polymorphisms K751Q and R438W in the case of XPD and POLL, respectively) for the genomic integrity and their direct roles in increased mutation rates potentially contributing to increased cancer susceptibility.

An interesting observation is that the genes POLL, BRCA1 and XPD represent the critical players of the three different DNA repair mechanisms of BER, NER and DSBR, respectively. In addition, none of these three proteins are in direct protein interaction with each other, where the latter two genes are linked to breast cancer through interaction with POLL. Therefore, these findings could suggest breast cancer risk in sporadic cases is more likely to be impacted when relatively distant but functionally complementary components of the general DNA re- pair processes are potentially impaired. This is in agreement with other studies that have demonstrated the critical role of interaction among distant biological systems, such as in yeast [44].

Despite these interesting results, some caution should be taken when interpreting those preliminary findings. Indeed, this study includes only one single SNP marker from the genomic region of each gene, thus misrepresenting the role of the entire gene sequence in breast cancer development. Furthermore, only a small number of genes $(\mathrm{n}=13)$ was included from the large cellular DNA repair pathway members ( $n \geq 100$ genes), thus giving a truncated picture of the overall activity of the DNA repair mechanism. Further work is therefore required in order to better evaluate the biological relevance of our results.

\section{Acknowledgements}

We would like to thank Dr. Irene Andrulis, Principal Investigator of the OFBCR for giving us access to the OFBCR data. This study was supported by Discovery Grant No. 293270-04 from the Natural Sciences and Engineering Research Council of Canada, the Samuel Lunenfeld Research Institute New Discoveries Funds, the McLaughlin Centre for Molecular Medicine (to S.K., L.B.), and the Canadian Breast Cancer Foundation (to H.O.). This study was also funded by the United States National Cancer Institute, National Institutes of Health, under Request for Application CA-95003 as part of the Breast Cancer Family Registries (CFRs) through a cooperative agreement with Cancer Care Ontario. The content of this paper does not necessarily reflect the views or policies of the National Cancer Institute or any of the collaborating centers in the CFRs nor does mention of trade names, commercial products, or organizations imply endorsement by the US Government or CFRs.

\section{References}

1 Friedman N: Inferring cellular networks using probabilistic graphical models. Science 2004;6:799-805.

2 Onay V, Briollais L, Knight J, Shi E, Wang Y, Wells S, Li H, Rajendram I, Andrulis I, Ozcelik H: SNP-SNP interactions in breast cancer susceptibility. BMJ Cancer 2006;6:114.

- 3 Taylor I, Linding R, Warde-Farley D, Liu Y, Pesquita C, Faria D, Bull S, Pawson T, Morris Q, Wrana J: Dynamic modularity in protein interaction networks predicts breast cancer outcome. Nat Biotech 2009;27:199-204.

4 Elston RC, Stewart J: A general model for the genetic analysis of pedigree data. Hum Hered 1971;21:523-542.

5 Thompson EA, Cannings C, Skolnick MH: Ancestral inference I. The problem and the method. Ann Hum Genet 1978;32:445-452.
6 Lauritzen SL, Sheehan NA: Graphical models for genetic analyses. Stat Sci 2003;18:489-514.

7 Thomas A, Gutin A, Abkevich V, Bansal A: Multipoint linkage analysis by blocked Gibbs sampling. Stat Comput 2000;10:259-269.

$>8$ Heath S, Robledo R, Beggs W, Feola G, Parodo C, Rinaldi A, Contu L, Dana D, Stambolian D, Siniscalco M: A novel approach to search for identity by descent in small samples of patients and controls from the same Mendelian breeding unit: a pilot study in myopia. Hum Hered 2001;52:183-190.

-9 George AW, Thompson EA: Discovering disease genes: Multipoint linkage analysis via a new Markov chain Monte Carlo approach. Stat Sci 2003;18:515-531.
10 Thomas A, Camp NJ: Graphical modelling of the joint distribution of alleles at associated loci. Am J Hum Genet 2004;74:1088-1101.

11 Verzilli CJ, Stallard N, Whittaker JC: Bayesian graphical models for genomewide association studies. Am J Hum Genet 2006;79:100-112.

12 Madigan D, York J, Allard D: Bayesian graphical models for discrete data. Intern Stat Rev 1995:63:225-232.

- 13 Xing $\mathrm{H}$, McDonagh PD, Bienkowska J, Cashorali T, Runge K, Miller RE, Decaprio D, Church B, Roubenoff R, Khalil IG, Carulli J: Causal modeling using network ensemble simulations of genetic and gene expression data predicts genes involved in rheumatoid arthritis. PLoS Comput Biol 2011; 7:e1001105. 
14 Ungvari I, Hullam G, Antal P, Kiszel PS, Gezsi A, Hadadi É, Virag V, Hajós G, Millinghoffer A, Nagy A, Kiss A, Semsei AF, Temesi G, Melegh B, Kisfali P, Széll M, Bikov A, Gálffy G, Tamasi L, Falus A, Szalai C: Evaluation of a partial genome screening of two asthma susceptibility regions using bayesian network based bayesian multilevel analysis of relevance. PLoS One 2012;7:e33573.

15 Jiang X, Barmada MM, Visweswaran S: Identifying genetic interactions in genome-wide data using Bayesian networks. Genet Epidemiol 2010;34:575-581.

16 Han B, Park M, Chen XW: A Markov blanketbased method for detecting causal SNPs in GWAS. BMC Bioinform 2010;11:S5.

$\checkmark 17$ Loeb KR, Loeb LA: Significance of multiple mutations in cancer. Carcinogenesis 2000;21: 279-385.

$\checkmark 18$ Moses RE: DNA damage processing defects and disease. Annu Rev Genomics Hum Genet 2001;2:41-68.

19 Berstein C, Berstein H, Payne CM, Garewal H: DNA repair/pro-apoptotic dual-role proteins in five major DNA repair pathways: failsafe protection against carcinogenesis. Mutat Res 2002;511:145-178.

20 Yu Z, Chen J, Ford BN, Brackley ME, Glickman BW: Human DNA repair systems: an overview. Environ Mol Carcinogen 1999;33: 3-20.

21 Mohrenweiser HW, Wilson DM 3rd, Jones IM: Challenges and complexities in estimating both the functional impact and the disease risk associated with the extensive genetic variation in human DNA repair genes. Mutat Res 2003;526:93-125.

22 Grossman L, Matanoski G, Farmer E, Hedayati M, Ray S, Trock B, Hanfelt J, Roush G, Berwick M, Hu JJ: DNA repair as susceptibility factor in chronic diseases in human populations; in Dizdaroglu M, Karakaya AE (eds): Advances in DNA Damage and Repair. New York, Kluwer, 1999, pp 149-167.
3 Berwick M, Vineis P: Markers of DNA repair and susceptibility to cancer in humans: an epidemiologic review. J Natl Canc Inst 2000;92: 874-897.

24 Edwards D: Introduction to Graphical Modeling. New York, Springer, 2000.

25 Lauritzen SL: Graphical Models. Oxford, Clarendon Press, 1996.

26 Dawid AP, Lauritzen SL: Hyper-Markov laws in the statistical analysis of decomposable graphical models. Ann Stat 1993;21:12721317.

27 Tarantola C: MCMC model determination for discrete graphical models. Stat Modelling 2004;4:39-61.

28 Madigan D, Raftery A: Model selection and accounting for model uncertainty in graphical models using Occam's window. J Am Stat Assoc 1994;89:1535-1546.

29 Cooper GF, Herskovits E: A Bayesian method for the induction of probabilistic networks from data. Mach Learn 1992;9:309-347.

30 Friedman N, Koller D: Being Bayesian about network structure. Mach Learn 2003;50:95125.

31 Green PJ: Reversible Jump Markov chain Monte Carlo computation and Bayesian model determination. Biometrika 1995;82: 711-732.

32 Dellaportas P, Foster JJ: Markov Chain Monte Carlo model determination for hierarchical and graphical log-linear models. Biometrika 1999;86:615-633.

33 Lunn DJ, Best N, Whittaker J: Generic reversible jump MCMC using graphical models. Stat Comput 2009;19:395-408.

-34 Sutherland HJ, Lacroix J, Knight J, Andrulis IL, Boyd NF; Ontario Cancer Genetics Network: The Cooperative Familial Registry for Breast Cancer Studies: design and first year recruitment rates in Ontario. J Clin Epidemiol 2001;54:93-98.
35 Yamane A, Kohno T, Ito K, Sunaga N, Aoki K, Yoshimura K, Murakami H, Nojima Y, Yokota J: Differential ability of polymorphic OGG1 proteins to suppress mutagenesis induced by 8-hydroxyguanine in human cell in vivo. Carcinogenesis 2004;25:1689-1694.

36 Donehower LA: p53: guardian AND suppressor of longevity? Exp Gerontol 2005;40:7-9.

37 Takanami T, Nakamura J, Kubota Y, Horiuchi S: The Arg280His polymorphism in X-ray repair cross-complementing gene 1 impairs DNA repair ability. Mutat Res 2005;582:135145.

38 Ramensky V, Bork P, Sunyaev S: Human nonsynonymous SNPs: server and survey. Nucleic Acids Res 2002;30:3894-3900.

39 Husmeier D: Sensitivity and specificity of inferring genetic regulatory interactions from microarray experiments with dynamic Bayesian networks. Bioinformatics 2003;19:22712282.

40 Costanzo M, Baryshnikova A, Bellay J, et al: The genetic landscape of a cell. Science 2010; 327:425-431.

41 Dobra A, Briollais L, Jarjanazi H, Ozcelik H, Massam H: Applications of the mode oriented stochastic search (MOSS) algorithm for discrete multi-way data to genomewide studies; in Dey D, Ghosh S, Mallick B (eds): Bayesian Modeling in Bioinformatics. Baco Raton, Taylor \& Francis, 2010, pp 63-93.

-42 Terrados G, Capp JP, Canitrot Y, García-Díaz M, Bebenek K, Kirchhoff T, Villanueva A, Boudsocq F, Bergoglio V, Cazaux C, Kunkel TA, Hoffmann JS, Blanco L: Characterization of a natural mutator variant of human DNA polymerase lambda which promotes chromosomal instability by compromising NHEJ. PLoS One 2009;4:e7290.

43 Symington LS, Gautier J: Double-strand break end resection and repair pathway choice. Annu Rev Genet 2011;45:247-271.

44 Boone C, Bussey H, Andrews BJ: Exploring genetic interactions and networks with yeast. Nat Rev 2007;8:437-449. 